\title{
A patient with abdominal pain following routine colonoscopy
}

\author{
Lorenz Theilmann ${ }^{1}$, Ulrich Voehringer ${ }^{2}$ and Ahmed Abdel Samie ${ }^{3 *}$ \\ ${ }^{1}$ Department of Gastroenterology, Helios Hospital, Pforzheim, Germany, substantial contributions to conception and design, acquisition of data \\ ${ }^{2}$ Department of Gastroenterology, Helios Hospital, Pforzheim, Germany, acquisition of data \\ ${ }^{3}$ Department of Gastroenterology, Department of Gastroenterology, Helios Hospital, Pforzheim, Germany, substantial contributions to conception and design
}

\begin{abstract}
Herein we report a case of splenic rupture following routine colonoscopy.

Splenic injury after colonoscopy is extremely rare, however internists/gastroenterologists should be aware of this possible potentially fatal complication and its delayed and unspecific onset.
\end{abstract}

\section{Case presentation}

A 44 years old female was admitted to the hospital with Crohn's disease involving the upper GI tract as well as the terminal ileum.

Diagnosis had been attained 4 months before histologically by gastroduodenoscopy and MRI enterography and treatment with corticosteroids had been initiated.

The patient was readmitted to complete staging including ileocolonoscopy which she previously had refused. Ileocolonoscopy was performed under conscious sedation using propofol and revealed active Crohn's disease of the distal ileum on a length of $10 \mathrm{~cm}$. The entire colon showed normal appearance. These findings were confirmed by histology.

The endoscopic procedure was uneventful. Progression of the scope up to the terminal ileum was achieved smoothly with no significant looping with a total procedure time of 12 minutes and withdrawal time of 8 minutes. The patient was asymptomatic and mobile following the procedure.

However, 10 hours later she reported slight dizziness and unspecific abdominal discomfort. During the following night she complained about abdominal pain. Lab tests showed a drop of her hemoglobin to $7.4 \mathrm{~g} / \mathrm{dl}$. Ultrasound examination was performed demonstrating pathological findings in the left upper abdomen (Figure 1, Figure 2).

Abdominal ultrasound revealed an enlarged spleen with hypoechoic areas at the upper splenic pole consistent with intrasplenic bleeding (Figure 1). In addition, free fluid was detected in the perisplenic abdominal cavity (Figure 2).

Diagnostic aspiration of this fluid confirmed Intraabdominal bleeding due to splenic injury. The patient was immediately transferred to the operating room, received transfusion of two packed red blood cells, and explorative laparotomy and splenectomy have been performed.

Intraoperatively pronounced adhesions of the greater omentum (mainly in the left lower abdomen) have been documented.

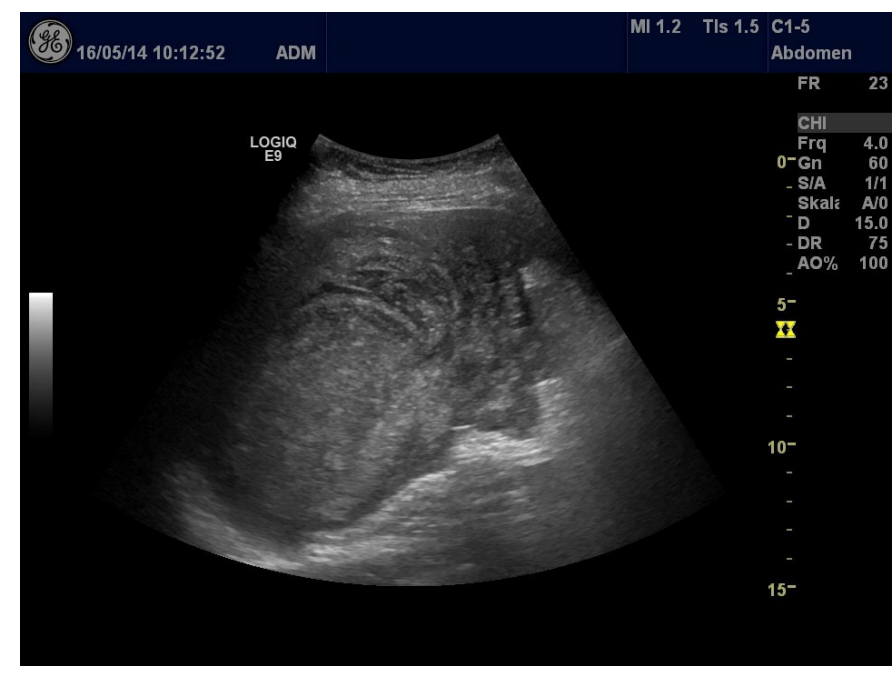

Figure 1. Ultrasound examination was performed demonstrating pathological findings in the left upper abdomen

The spleen was partially avulsed with subcapsular hematoma and secondary rupture. The postoperative course was uneventful, and the patient was discharged after five days having received triple vaccination against postsplenectomy infections according to the current guidelines. Treatment of Crohn's disease was resumed.

\section{Discussion}

Splenic injury due to colonoscopy is a rare complication and most patients have delayed symptoms. Less than 80 cases have been reported so far.

${ }^{*}$ Correspondence to: Ahmed Abdel Samie, Helios Hospital, Pforzheim Germany, Kanzlerstrasse, Pforzheim Germany, Tel: + 49 1736923849; E-mail: abdelsamie@ngi.de

Received: May 11, 2018; Accepted: May 23, 2018; Published: May 28, 2018 


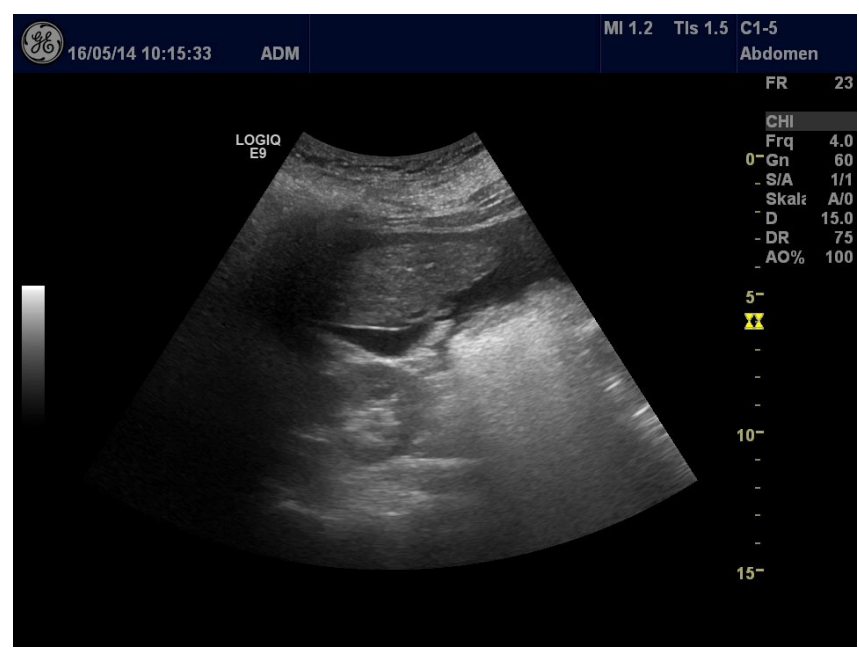

Figure 2. Ultrasound examination was performed demonstrating pathological findings in the left upper abdomen

In systematic reviews $[1,2]$ two hypothetic mechanism of trauma have been suggested. Direct trauma by the endoscope being positioned in the left flexure, yet this mechanism of injury appears to be less frequent than excessive traction on the splenocolic ligament when advancing the endoscope into the transverse colon leading to avulsion of the splenic capsule [1].
In most cases so far, adhesions by previous surgery or inflammatory processes have been reported. Symptoms usually start 24 hours after colonoscopy when significant amount of blood accumulates in the subcapsular space [3] but can be delayed for several days. Most patients present with hemorrhagic shock at diagnosis.

FAST (focus assessment with sonography for trauma) or contrast enhanced CT scan are the diagnostic tools of choice [3]. Although endovascular treatment of splenic bleeding has been reported, a positive FAST in the presence of hemodynamic instability should lead to immediate explorative laparotomy.

Splenic injury after colonoscopy is extremely rare, however endoscopists should be aware of this possible potentially fatal complication and its delayed and unspecific onset.

\section{References}

1. Corcillo A, Aellen S, Zingg T, Bize P, Demartines N, et al. (2013) Endovascular treatment of active splenic bleeding after colonoscopy: a systematic review of the literature. Cardiovasc Intervent Radiol 36: 1270-1279. [Crossref]

2. Aubrey-Bassler FK, Sowers N (2012) 613 cases of splenic rupture without risk factors or previously diagnosed disease: a systematic review. BMC Emerg Med 12: 11. [Crossref]

3. Michetti CP, Smeltzer E, Fakhry SM (2010) Splenic injury due to colonoscopy: analysis of the world literature, a new case report, and recommendations for management. $\mathrm{Am}$ Surg 76: 1198-1204. [Crossref]

Copyright: (C2018 Theilmann L. This is an open-access article distributed under the terms of the Creative Commons Attribution License, which permits unrestricted use, distribution, and reproduction in any medium, provided the original author and source are credited. 